\title{
18. Substance use in the 2002 NATSISS
}

\section{Tanya Chikritzhs and Maggie Brady}

It is important at the outset to acknowledge with candour that questioning Aboriginal or Torres Strait Islander people about their use of alcohol and other drugs is always fraught with difficulty, whatever the circumstance. As Anderson and Sibthorpe (1996: 118-134) observed of the 1994 NATSIS, one wonders about the subjective meanings that might be attached to such questions, what are the perceptions of personal risk that might be attached to such questions, and what interviewees understand to be the purpose of such information. Questions of this sort can meet with resistance, underestimation and 'fudging' even if asked privately by a health professional and in an ostensibly 'safe' environment such as an Indigenous health service (Brady et al. 2002). Usually, health service providers will ask such questions only after having first put people at ease, opening the discussion on alcohol and other drugs by phrasing questions colloquially and/or by first having the results of preliminary screening to hand.

Reliable survey estimates of substance use that specifically address Indigenous populations are important for several reasons. They provide a measure of the prevalence of substance use among Indigenous people for males and females and for a range of ages; such information is not available from any other source. For example, Indigenous alcohol consumption cannot be identified from per capita alcohol consumption estimates. When surveys are conducted regularly and consistently, they can also be used to measure changes in use over time. Survey estimates of the population prevalence of tobacco, alcohol and other drug use are a vital component in the estimation of 'population aetiologic fractions' for these substances - the degree to which a substance can be said to cause various diseases and injuries in a certain population. Accurate prevalence estimates of alcohol consumption are therefore necessary for the estimation of alcohol-attributable deaths and hospitalisations. In turn, reliable estimates of levels of alcohol-related harms can be used to inform the allocation of funding and resources for prevention and treatment programs (Gray et al. 2002).

For a national survey to be of local relevance, it needs to:

- contain sufficient numbers of respondents from Indigenous populations throughout Australia

- include a broad range of ages and both sexes, and

- use appropriate methods for measuring drinking levels and patterns. 
It should be kept in mind that although alcohol survey methods have improved in recent years (Stockwell et al. 2004), when compared to per capita consumption estimates from wholesale data, past surveys have always dramatically underestimated alcohol consumption.

\section{Overview of the 2002 NATSISS methods in relation to substance use}

In their chapter to this volume, Biddle and Hunter describe the survey methods used by the 2002 NATSISS in detail. This section will briefly review those methods with a specific focus on the collection of information in relation to alcohol, tobacco and other substances (e.g. illicit substances). It is worth pointing out here that we use the term 'substance use' to include alcohol and tobacco use, as is the normal practice in the alcohol and other drugs area, whereas in the NATSISS 'substance use' seems to refer only to other drugs, including illicit drugs.

\section{Selecting a representative sample}

The 2002 NATSISS used distinctly different sets of methods for sampling Indigenous populations. These methods were not applied at random or equivalently throughout the Indigenous population of Australia but were purposely targeted at specific sub-populations largely relating to geographic location. Thus, the sampled population was effectively divided into two parts:

1. A random sample of households from discrete Indigenous communities and the outstations associated with them in areas of Queensland, South Australia, Western Australia and the Northern Territory. This was known as the CA sample. Although it is not entirely clear, the ABS has suggested that the majority of respondents sampled using this method were resident in very remote areas as distinct from remote areas (i.e. using ARIA classification) - see Biddle and Hunter in this volume. Approximately 23 per cent of the total 9359 respondents were sampled in this way.

2. The remaining respondents were resident in NCAs and were selected using a stratified multi-stage area sample based on the 2001 Census. A random selection of dwellings within selected census CDs was then screened to assess Indigenous status of usual residents. According to the ABS, an insufficient number of households with Indigenous residents were initially collected, so additional CDs were sampled during February to April 2003. The majority of respondents to the 2002 NATSISS were sampled using this method.

Those who lived in remote regions but not in discrete Indigenous communities were sampled using only the NCA method. 


\section{Asking about drug use in the 2002 NATSISS}

Two main methods were used for asking about drug use in the 2002 NATSISS:

- face-to-face interviewer questioning of the respondent requiring a verbal response, using either computer assisted or pen and paper methods, or

- voluntary, confidential (i.e. self-sealed), self-complete questionnaire.

The voluntary self-complete option was exclusively available to respondents of NCAs who agreed to answer questions on illicit drug use. For both alcohol and tobacco use, only face-to-face interviews were used. In addition, the self-complete questionnaire on illicit drug use was not available to residents living in CAs and these respondents were required to submit their responses verbally to an interviewer.

\section{Tobacco}

From the non-remote areas questionnaire, it can be ascertained that three questions were asked about tobacco use during face-to-face interviews. They were:

- Do you currently smoke?

- Do you smoke regularly, that is, one smoke a day or more?

- Have you ever smoked regularly (that is, one smoke a day or more)?

Residents of CAs were similarly asked about smoking behaviour.

\section{Alcohol}

The alcohol questions used in the 2002 NATSISS were different from those of the 1994 NATSIS. For the 2002 survey, respondents were asked to estimate how often they drank in the last year and the quantity and type of alcohol they usually consumed on a drinking day. These were later converted to standard drinks by the interviewer. The quantity/frequency method is adequate for estimating rates of abstainers but is known to result in underestimation of the volume of alcohol people drink per occasion (Stockwell et al. 2004).

\section{Illicit drug use}

The 2002 NATSISS asked respondents about their use of substances other than alcohol and tobacco, referring to this group of drugs as 'substance use'. The 'substance use' questions included the use of a range of drugs for non-medical purposes and were based on questions used in the NDSHSs. In the survey, 'substance use' included analgesics, tranquillisers, amphetamines, marijuana, heroin, cocaine, hallucinogens (both synthetic and naturally occurring), ecstasy and other designer drugs, petrol and other inhalants, and kava. Respondents were asked to report on their use in the last 12 months or if they had ever used any of these substances. Unfortunately, however, according to the ABS (2004c), 
information on substance use will not be released for residents of remote areas 'due to data quality concerns' (see p.79), largely arising from the manner in which the questions were asked (i.e. face-to-face interview requiring a verbal answer). In effect, therefore, the findings of the 2002 NATSISS in relation to illicit drug use are limited to non-remote populations and cannot be used to inform overall levels of use among Indigenous people (since use in over $20 \%$ of the sample is not accounted for).

\section{Estimates of Indigenous drug use from the 2002 NATSISS}

The results of the 2002 NATSISS in relation to substance use (i.e. tobacco, alcohol and illicit drugs) have been summarised by the ABS (2004c).

Table 18.1. Drug use among Indigenous Australians: results from the 2002 NATSISS

\begin{tabular}{|c|c|c|c|}
\hline \multirow[t]{2}{*}{ Risk behaviour/characteristics } & Remote & Non-remote & Total \\
\hline & $\%$ & $\%$ & $\%$ \\
\hline \multicolumn{4}{|l|}{ Smoker status } \\
\hline Current daily smoker & 50.4 & 48.0 & 48.6 \\
\hline Occasional smoker & 2.9 & 2.0 & 2.3 \\
\hline Ex-smoker & 11.5 & 16.7 & 15.3 \\
\hline Never smoked & 32.7 & 33.3 & 33.2 \\
\hline \multicolumn{4}{|l|}{ Alcohol consumption level in last 12 months } \\
\hline Low risk & 32.2 & 51.3 & 46.1 \\
\hline Risky & 10.0 & 9.4 & 9.6 \\
\hline High risk & 6.8 & 5.1 & 5.6 \\
\hline Any alcohol use & 53.6 & 75.3 & 69.4 \\
\hline Did not consume alcohol in last 12 months & 46.4 & 24.7 & 30.6 \\
\hline \multicolumn{4}{|l|}{ Type of substance used in last 12 months ${ }^{a}$} \\
\hline \multicolumn{4}{|l|}{ Used substances in last 12 months } \\
\hline Analgesics and sedatives for non-medical use & - & 4.4 & - \\
\hline Amphetamines or speed & - & 4.7 & - \\
\hline Marijuana, hashish or cannabis resin & - & 19.1 & - \\
\hline Kava & - & 0.6 & - \\
\hline Total used substances in last 12 months & - & 23.5 & - \\
\hline
\end{tabular}

a. Respondents may have indicated more than one response per category. Data only available for non-remote areas. 'Substances' includes heroin, cocaine, hallucinogens, designer drugs, petrol and other inhalants. Source: ABS (2004c: 40)

According to the 2002 NATSISS, almost one half of Indigenous respondents were current daily smokers. In comparison, non-Indigenous current tobacco use has been estimated by the 1998 and 2001 National Drug Strategy Household Surveys to be around 25 per cent and 23 per cent respectively-less than half that for the Indigenous population (Higgins, Cooper-Stanbury \& Williams 2000). ${ }^{1}$

\footnotetext{
1 According to the 2004 NDSHS, smoking rates among the non-Indigenous population have declined since 2001 (AIHW 2005).
} 
The ABS identified a 90 per cent response rate to its self-complete 'substance use' questionnaire (administered in NCAs but not CAs). However, as discussed earlier, the ABS has only reported on illicit substance use by respondents who resided in NCAs. With this qualification in mind, 19.1 per cent of Indigenous respondents claimed to have used cannabis in the previous 12 months. The 2001 NDSHS has estimated that among the non-Indigenous population, cannabis use in the last 12 months was about 13 per cent (AIHW 2002). More Indigenous respondents reported having used cannabis in the last 12 months than any other illicit substance - including amphetamines (4.7\%) and kava $(0.6 \%)$. Less than 5 per cent of respondents reported having used analgesics or sedatives for non-medicinal purposes.

In relation to the proportion of respondents who did or did not use 'substances' in the last 12 months, the summary table given in the ABS report presents some conflicting results. The last two lines of the table show: 'Total used substances in last 12 months' and 'Did not use substance in last 12 months', the proportions for which are given as 23.5 per cent and 16.2 per cent respectively. These two figures are irreconcilable, and it would appear that an error has been made in at least one of these totals. ${ }^{2}$

According to the 2002 NATSISS, 30.6 per cent of all Indigenous participants reported being abstinent from alcohol in the past 12 months. Respondents who lived in remote regions were almost twice as likely as those living in non-remote areas to report no alcohol consumption in the previous 12 months (46.4\% versus $24.7 \%$ ). In addition, 46.1 per cent of all respondents reportedly drank at levels that placed them at low risk for alcohol related harms. Of the remaining 23.3 per cent of the sample, 15.2 per cent were reported as having drunk at levels that placed them at risky or high risk for alcohol related harm. ${ }^{3}$ However, a substantial proportion of the sample $(8.1 \%)$ was not accounted for in the summary of drinking patterns provided in the ABS (2004c) (i.e. levels reported on p.40 do not add up to $100 \%$ ). It is possible that these respondents either refused to respond to questions about alcohol use or they gave responses that were problematic.

Previous reviews that have compared Indigenous and non-Indigenous alcohol use in Australia have found that although Indigenous people are more likely to abstain from alcohol, they are also more likely to drink at risky/high-risk levels for alcohol-related harm than their non-Indigenous counterparts (e.g. Gray et al. 2004). The 2001 NDSHS estimated that among the non-Indigenous population Australia-wide, 17.8 per cent abstained from drinking alcohol (i.e. had not drunk

2 The ABS confirmed that the summary table is subject to typographical error at CAEPR conference and that these values are incorrect. We await further information from the ABS.

3 It is not clear from the ABS report whether the drinking patterns reported were in relation to the latest Australian drinking guidelines for acute or chronic harms (i.e. NHMRC 2001) or a composite of both. 
in the last 12 months) - about half that estimated for the Indigenous population by the 2002 NATSISS. In addition, some 71.7 per cent of non-Indigenous respondents reported drinking at low-risk levels for alcohol-related harms, while the remaining 10.4 per cent drank at risky or high-risk levels. ${ }^{4}$ The recently released results of the 2004 NDSHS confirm that levels of drinking have remained relatively stable among the non-Indigenous population (AIHW 2005b). In summary, therefore, according to the results of the 2002 NATSISS and the 2001 NDSHS, the comparative discrepancy between Indigenous and non-Indigenous risky/high-risk use of alcohol in recent years was less than 5 per cent (see Fig. 18.1).

Figure 18.1. A comparison of Indigenous and non-Indigenous drinking levels, from the 2002 NATSISS and the 2001 NDSHS

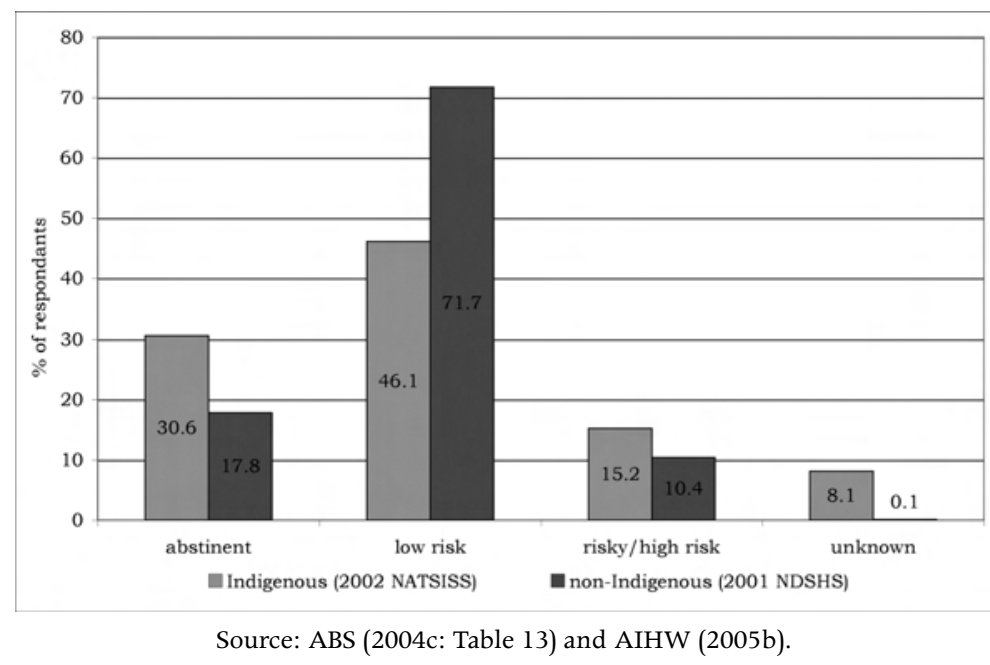

The small apparent difference between Indigenous and non-Indigenous risky/high-risk alcohol consumption suggested by the 2002 NATSISS results is particularly surprising given recent comparisons of alcohol-attributable hospitalisations for the two populations. For example, data cited by the SCRGSP (2005) noted that in 2002-03 the rate of hospital admission among Indigenous males was between two and seven times greater than for non-Indigenous males (see Table 18.2). This was for conditions related to high levels of alcohol use, such as acute alcohol intoxication, alcoholic liver disease, harmful use, and alcohol dependence.

4 These summaries are not available in the AIHW detailed findings report. They were calculated by the National Alcohol Indicators Project for the 2003 report on Australian Alcohol Indicators (Chikritzhs et al. 2003). 
Table 18.2. Indigenous and non-Indigenous rates of hospitalisation (per 1000) for a selection of alcohol-attributable conditions 2002-03

\begin{tabular}{lrrr}
\hline Condition & $\begin{array}{r}\text { Indigenous rate } \\
\%\end{array}$ & $\begin{array}{r}\text { Non-Indigenous rate } \\
\%\end{array}$ & Rate ratio \\
\hline Acute intoxication & 3.5 & 0.5 & 7.0 \\
Alcohol dependence & 2.4 & 1.0 & 2.4 \\
Alcoholic liver disease & 1.5 & 0.3 & 5.0 \\
Harmful use & 0.4 & 0.1 & 4.0 \\
\hline
\end{tabular}

Source: SCRGSP (2005: Table 8A.2.2)

Other studies have shown that the rate of death from wholly alcohol-caused conditions (e.g. alcoholic liver cirrhosis, alcohol dependence) among residents of WA, SA and the NT is almost eight times greater for Indigenous males than for non-Indigenous males and 16 times greater for Indigenous females (Chikritzhs et al. 2000). The level of alcohol-attributable death among young Indigenous Australians (aged 15-24 years) has also been shown to be almost three times greater than for their non-Indigenous counterparts - with the divergence between the two populations apparently increasing in recent years (Chikritzhs \& Pascal 2004). The Indigenous National Alcohol Indicators Project has also estimated the crude rate of alcohol-attributable deaths among Indigenous Australians versus the general population from 1999 to 2002. ${ }^{5}$ As shown in Figure 18.2, the rate of death from alcohol-attributable conditions is over two and a half times greater for Indigenous people than for the general population, and the relative proportions have not changed substantially in recent years.

${ }^{5}$ Alcohol-attributable crude death rates have been derived from the data held by the Indigenous National Alcohol Indicators Project at the National Drug Research Institute, and have been specifically produced for this report. Age standardised rates are not yet available. 
Figure 18.2. Estimated national alcohol-attributable death rates for Indigenous people versus the general Australian population

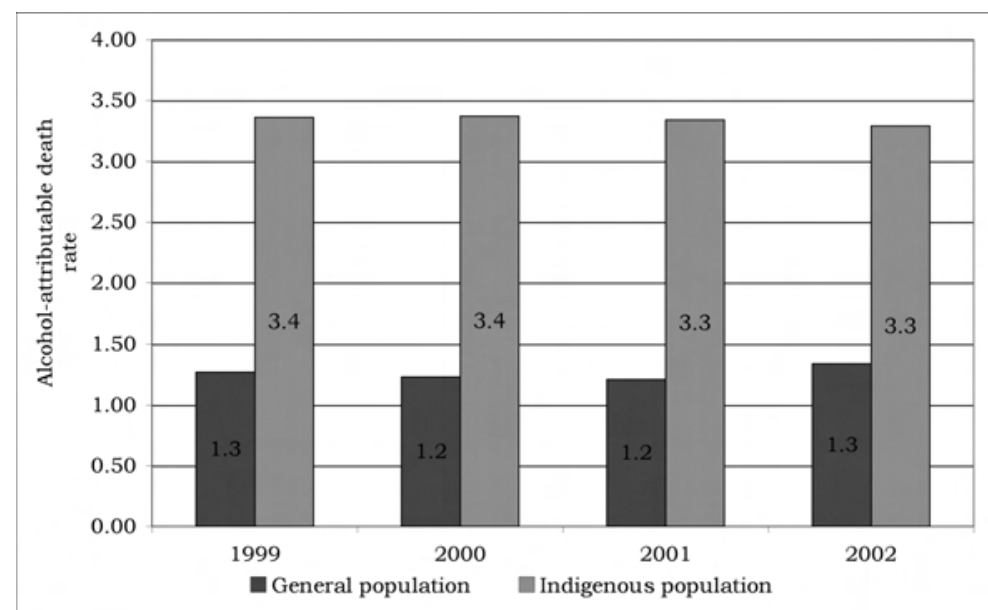

Source: Customised cross-tabulations from the National Alcohol Indicators Project alcohol-attributable mortality database, National Drug Research Institute, Perth.

\section{How do the 2002 NATSISS results compare with other national surveys of Indigenous drug use?}

To date, only five national surveys with moderate to large sample sizes have specifically addressed Indigenous alcohol consumption throughout Australia. They include the:

- 1994 National Drug Strategy Household Survey Urban Aboriginal and Torres Strait Islander Peoples Supplement (NDSHS) conducted by AGB McNair on behalf of the Commonwealth Department of Human Services and Health (CDHSH 1996)

- 1994 NATSIS (ABS, 1995b)

- 1995 NHS (ABS 1999)

- 2001 NHS (ABS 2002a), and

- 2002 NATSISS (ABS 2004c).

The latter four surveys were conducted by the ABS. A range of small local surveys have also been conducted but they are generally not suitable for comparisons between regions (see Saggers \& Gray 1998). This section compares the results of the 2002 NATSISS with those derived for the Indigenous population by the 1994 NATSIS, the 2001 NHS and the 1994 NDSHS.

\section{NATSIS}

The most obvious starting point for comparison with the 2002 NATSISS outcomes for substance use is the 1994 NATSIS. Unfortunately, the use of incompatible methods and different questions applied in the two surveys has limited the 
comparisons that can be drawn between them. The ABS has limited its own tabulated comparisons to estimates of current smokers and the proportion of respondents who drank alcohol in the past 12 months, with no comparisons possible for illicit substances.

Despite sampling differences, the two surveys estimated similar levels of current smokers - 51.7 per cent in 1994 and 50.9 per cent in 2002. Likewise, the estimated proportions of Indigenous people who abstained from alcohol in the last 12 months were 32.4 per cent in 1994 and 30.6 per cent in 2002. It is not possible however, to compare the proportion of drinkers consuming alcohol at various levels (i.e. low, risky, high risk) between these two surveys. In summary, although the comparisons are severely limited, there is no strong evidence of change over time in tobacco and alcohol use - on these two measures, at least.

\section{The 2001 NHS}

It is also useful to compare the 2002 NATSISS with the 2001 NHS (ABS 2002b). Given the temporal proximity of these two surveys - and if both had adequately and reliably measured drug use - then all other things being equal, we would expect to find similar national estimates. ${ }^{6}$

The 2001 NHS included respondents from both remote and non-remote areas of Australia but did not include an especially large number of respondents (1853 adults) (ABS 2002b). (Indeed, due to the small sample size, the ABS limited the scope of all publications to reporting at a national level.) Nevertheless, among Indigenous respondents, the estimated proportion of current smokers from the 2001 NHS was about 51 per cent - a few points higher than that estimated by the 2002 NATSISS $(48.6 \%)$.

With regard to patterns of alcohol use, the method used by the 2001 NHS was especially problematic. Although a quantity-frequency method was applied for measuring alcohol consumption, the survey only asked about drinking that had occurred in the week before the interview (those who did not drink in the previous week recorded no alcohol consumption). What is more, only details of the three most recent drinking days during that week were recorded. The shortcomings of this method have been well documented elsewhere (e.g. Rehm et al. 1999; Stockwell et al. 2004; WHO 2000)-especially in relation to underestimated levels of consumption and numbers of episodic drinkers (i.e. binge drinkers). In particular, since the drinking patterns of a large proportion of Indigenous drinkers tend to be intermittent and clustered around fortnightly payment of social security entitlements, the 'three day method' used was particularly prone to underestimation of total alcohol consumption and ill-equipped to measure Indigenous alcohol consumption.

\footnotetext{
6 The 2001 NHS did not ask Indigenous respondents about illicit drug use.
} 
Keeping in mind the shortcomings of the survey method applied in the 2001 NHS, it was reported by the ABS that 42 per cent of Indigenous respondents drank in the week before the interview ( $58 \%$ were abstinent in the previous week) and 29 per cent of those who drank consumed alcohol at risky or high-risk levels (ABS, 2001a). From this we can deduce that 12 per cent of all Indigenous respondents drank at risky/high-risk levels, while the remaining 30 per cent drank at low-risk levels in the previous week. ${ }^{7}$

\section{NDSHS Urban Aboriginal and Torres Strait Islander Peoples Supplement}

The only major population survey that has focused specifically on substance use among Indigenous Australians is the 1994 NDSHS Urban Aboriginal and Torres Strait Islander Peoples Supplement (CDHSH 1996). ${ }^{8}$ Despite its age, this survey is arguably the most reliable to date (Gray et al. 2004). Indeed, a comparison between the 1994 NDSHS and the 2002 NATSISS is perhaps the most revealing measure of the shortcomings of the latter survey.

The 1994 NDSHS was conducted in response to recommendations by the Royal Commission into Aboriginal Deaths in Custody. It was conducted by AGB McNair on behalf of the then Commonwealth Department of Human Services and Health. As part of the survey, face-to-face interviews were conducted with 2993 Indigenous people aged 14 years and older residing in 'urban centres' of Australia. Urban centres were defined as either 'major' or 'other' but were required to include a minimum of 1000 people for inclusion in the survey (the majority of Indigenous Australians reside in such centres) (CDHSH 1996).

The 1994 NDSHS estimated that about 54 per cent of respondents were 'current' smokers (i.e. smoked in the previous 12 months) - about 4 percentage points higher than that reported by both the 2002 NATSISS (48.6\%) and the 2001 NHS $(51 \%)$. This may indicate a possible reduction in the proportion of Indigenous smokers over the period between surveys. Others have also noted that apparent declines had occurred in the proportion of current smokers between 1994 and 1998 (Gray et al. 2004). In relation to those who have 'ever smoked', there was also an apparent decline, with the 1994 NDSHS estimating about 77 per cent of the sample as having ever smoked, compared to about 67 per cent from the 2002 NATSISS. Nevertheless, the prevalence of smoking among Indigenous people remains about twice as high as that for non-Indigenous people (Gray et al. 2004).

\footnotetext{
7 Although questions about drinking in the last two weeks were asked by the 2002 NATSISS, concerns regarding the methodological approach have restricted publication of the results. However, personal communication with the ABS has identified that the proportion of respondents reporting risky/high-risk use in the last two weeks of the 2002 NATSISS was substantially higher than the proportion of respondents reporting the same level of drinking in the last 12 months.

8 With the exception of the - as yet- unpublished 2004 NHS.
} 
Some 48 per cent of Indigenous participants in the 1994 NDSHS reported having ever used cannabis and 22 per cent had used in the last 12 months. According to the 2002 NATSISS, about 19 per cent of respondents residing in NCAs had used cannabis in the previous 12 months. In the 1994 NDSHS, 19 per cent of Indigenous participants reported using an illicit drug other than cannabis. The summary of results from the 2002 NATSISS regarding 'substance use' indicates that the overall estimated proportion of residents in NCAs that had used a substance in the last 12 months was 23.5 per cent (ABS 2004c). Nineteen per cent had used cannabis in the last 12 months and a total of 9.7 per cent had used other drugs listed in the report (i.e. analgesics, amphetamines, sedatives, kava). Given this, it would appear (although it is difficult to tell definitively from the report because not all drugs were listed) that somewhere in the order of 10 per cent of respondents had used drugs other than cannabis in the recent past. This is a much smaller proportion than that estimated by the 1994 NDSHS. However, comparisons between the two surveys - and therefore any consideration of changes over time - are complicated by the fact that the 2002 NATSISS findings in relation to illicit drug use are specifically limited to non-remote Indigenous populations. In addition, it is worth noting that the standard errors in relation to reported kava use were considerable (between 25\% and 50\%) (ABS 2004c).

The 1994 NDSHS used a standard quantity-frequency method for estimating 'usual' alcohol consumption - as did the 2002 NATSISS. This method is adequate for estimating rates of abstainers but will result in underestimation of the volume of alcohol people drink per occasion (Stockwell et al. 2004). Most importantly, questions about alcohol in the 1994 NDSHS were contained in a confidential sealed section for self-completion by the respondent, so no direct questioning from the interviewer was required.

In relation to alcohol, results from the 1994 NDSHS showed that about 38 per cent of Indigenous people living in urban centres had not drunk alcohol in the previous year, compared to 28 per cent of the general population (CDHSH 1996). Thus, Indigenous people were more likely to be non-drinkers than members of the general population. In fact, the 1994 NDSHS reported a greater proportion of Indigenous respondents as being abstinent in the last 12 months than did the 2002 NATSISS (30.6\%). Nevertheless, based on amounts usually consumed, the 1994 NDSHS estimated that 82 per cent of all Indigenous current drinkers were considered at risk or high risk compared to 28 per cent of non-Indigenous drinkers (CDHSH 1996). Overall, therefore, an estimated 51 per cent of all Indigenous respondents to the 1994 NDSHS were considered to be drinking at risky or high-risk levels for alcohol-related harm-over three times greater than that estimated by the 2002 NATSISS. Reported levels of alcohol consumption from the 2002 NATSISS, 2001 NHS and 1994 NDSHS have been summarised in Figure 18.3. 
Figure 18.3. Estimates of alcohol use by Indigenous Australians: a comparison of three surveys

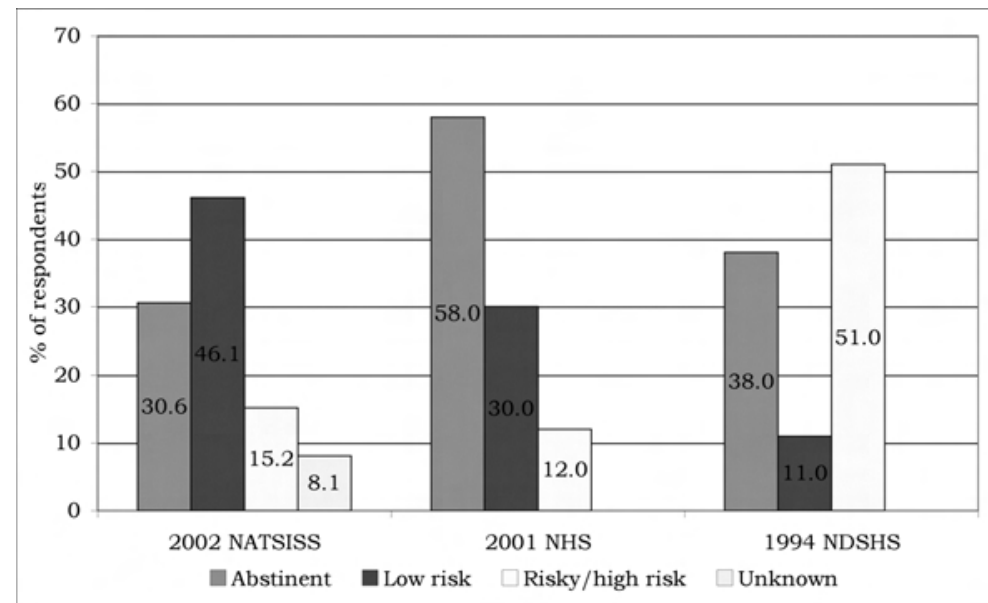

Source: ABS (2004c: Table 13) and CDHSH (1996).

Another surprising finding was that a higher percentage of Torres Strait Islander people than Aboriginal people were found to have risky or high-risk alcohol consumption (see Fig. 18.4).

Figure 18.4. 2002 NATSISS, Aboriginal and Torres Strait Islander risky/high-risk alcohol consumption

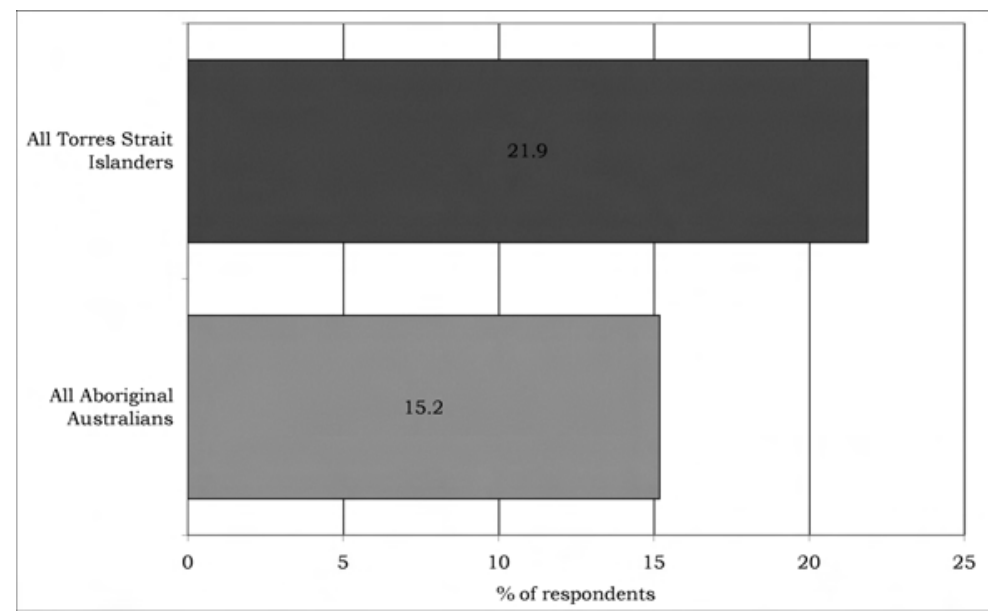

Source: ABS (2004c: Table 1).

\section{What can be said about the results of the 2002 NATSISS?}

All the surveys identified above have sought to inform on Indigenous substance use, yet they are all different and each of them has its limitations - some more serious than others. It is difficult to reliably compare results between surveys 
that do not have very similar methods, particularly where they vary in relation to sample selection, survey design and administration (e.g. questionnaire). Errors related to sampling can often be estimated and due consideration given to interpretation of the results. However, non-sampling error - such as may arise in the actual process of questioning respondents about their drug use - is less likely to be immediately obvious and the extent of it can rarely be measured. Variation between surveys also makes it difficult to determine whether differences which may appear over time or between populations are, in fact, real, rather than aberrations of the methods applied.

So, what can be said about the results of the 2002 NATSISS in relation to Indigenous drug use, and with what level of confidence? In short, this is a thorny question and one which is best served by examining some of the likely sources of error in relation to substance use from the NATSISS. These are discussed below.

\section{Exclusion of residents living in non-private dwellings}

Collecting information only from those living in private dwellings will arguably have a greater impact on responses to questions on the topic of alcohol and other drug use than on many other topics. Numerous Indigenous people living in hostels, short-stay caravan parks, prisons and other correctional facilities, hotels and hospitals were excluded. Alcohol-dependent people and problem drinkers in any population will be under-represented in household surveys, as Stockwell et al. (2004) have pointed out. They are more likely to be of no fixed abode, to be in an unfit state to participate, more likely to be incarcerated, and reluctant to discuss their drinking. Larson (1996) among others, has pointed out that many Aboriginal drug users (especially injectors) have insecure accommodation, are itinerant, or are living in supported accommodation and rehabilitation centres. One can conclude then, that the NATSISS would have missed numerous users of illicit drugs during the 2002 survey, as well as many problem drinkers for the same reasons.

\section{Insufficient data on drinking patterns}

Two 'big' questions that are important to alcohol researchers working on Indigenous data are the extent of drinking to the point of intoxication (i.e. binge drinking), and whether more people are becoming abstainers. Binge drinking is proving to be increasingly important as a predictor of physical harm, and is usually defined as drinking in order to get drunk in a short time. The number of drinks consumed per occasion is an important risk factor for death from injury, whereas frequency of consumption is not. There is a developing consensus (based largely on studies of alcohol-related mortality in the former Soviet Union) that repeated binge drinking is linked to sudden cardiac death, increased risk of thrombosis and high blood pressure (Hemstrom 2001; Room 2001). The 2002 
NATSISS asked 'when you drink, how much do you usually drink in a day', rather than 'how often do you have six or more drinks on one occasion', which is the preferred 'binge' question. On the second big question - that is, the proportion of individuals who had quit drinking - the 2002 NATSISS did not have a question on being an ex-drinker (although they did have a question on being an ex-smoker).

\section{Lack of confidentiality leading to unreliable responses}

Despite its lack of intrinsic appeal, the decision to avoid the use of self-completed questionnaires in CAs for illicit 'substance use' questions was prompted by results from pilot testing. According to the ABS, pilot testing indicated that poor literacy levels in these very remote areas posed significant problems in relation to the use of self-complete questionnaires. Unfortunately, however, the lack of confidentiality imposed on such a sensitive topic diminished the reliability of the responses to such an extent that it led the ABS to abandon the use of the information. The abandonment of these evidently problematic findings on illicit substances begs the question of whether the alcohol consumption data was similarly diminished in quality. It is perplexing that while questions on illicit substance use were considered too sensitive for face-to-face questioning in NCAs, despite the potential discomfiture, at no time were respondents given the opportunity to reply to questions on alcohol use in a confidential fashion. We believe that alcohol consumption is as sensitive a topic as illicit drug use.

The use of facilitators may also have resulted in varying degrees of error, although it is hard to predict the potential differential impact of questioning in the presence of, or without, an Indigenous facilitator. Whether the Indigenous facilitator is known to the respondent could influence responses. Social proximity to an Indigenous facilitator could result in the respondent minimising their consumption rates of alcohol and other drugs (Brady et al. 2002).

\section{Insensitivity to geographical and cultural diversity}

Surveys that include questions on illicit drug use should ideally be part of specialised national drug use surveys (rather than a general 'social' survey), such as the Australian Needle and Syringe Program surveys. Otherwise, they should be conducted regionally by less threatening organisations that have more intimate contact with the subjects. National surveys do not take into account geographical and cultural variations in the prevalence, patterns or availability of illicit drugs among so many diverse Aboriginal groups (Shoobridge 1997). They find that more regionally specific and targeted surveys using rapid assessment methodologies are of more use (Aboriginal Drug and Alcohol Council 2004).

The inclusion of a question on kava use is puzzling, as Indigenous users of this soporific drink (imported from the Pacific) are confined almost entirely to one region of the country (Arnhem Land), and probably number in the hundreds. 
No findings are available for CAs (remote areas) and the estimates for the non-remote sample are unreliable. This is unsurprising, as the use of kava by Indigenous Australians in these settings is unknown.

\section{Limited corroboration with other sources of information}

The ability to corroborate findings between various sources of information is indicative of the level of confidence that can be placed in the results of any particular study. In the case of the 2002 NATSISS and its findings on substance use, the corroborative evidence is weak.

Population estimates of risky/high-risk alcohol use drawn from the 2002 NATSISS indicate that about 15 per cent of the Indigenous population drink at risky/high-risk levels. This can be compared to other surveys which have estimated that about 10 per cent of the non-Indigenous population drink at the same level (AIHW 2002). This does not concur with the exceptionally high rate of death and hospitalisation from alcohol-attributable conditions which arise from risky/high-risk drinking (e.g. alcoholic liver cirrhosis, alcohol dependence) among the Indigenous compared to the non-Indigenous population. Indeed, the resultant drinking patterns from the 2002 NATSISS more closely resemble a non-Indigenous drinking pattern than any other previously conducted surveys of Indigenous drinking. That is, the majority of Indigenous respondents are consuming at low-risk levels; a smaller proportion are abstinent; and the smallest proportion drink at risky/high-risk levels. With the exception of the 2002 NATSISS, all of the national surveys described here indicated much larger proportions of Indigenous abstainers than low-risk drinkers. (Even the 2001 NHS, with all its problematic methodology, found a larger proportion of Indigenous abstainers than low-risk drinkers.)

Moreover, data on other alcohol-related issues, such as homicide, do not corroborate the suggestion in the 2002 NATSISS that a large proportion $(46 \%)$ of Indigenous drinkers are consuming alcohol at low-risk or relatively safe levels. For example, between 1999 and 2003, 71.3 per cent of Indigenous homicides occurred in situations where both perpetrator and victim were drinking (as opposed to $19.5 \%$ of non-Indigenous homicides) (SCRGSP 2005).

The 2002 NATSISS results are vastly dissimilar to the only national survey specifically relating to substance use conducted in Australia to date - and arguably the most reliable - the 1994 NDSHS. The 1994 NDSHS indicated over three times the number of risky/high-risk drinkers than did the NATSISS. Given that all surveys of drinking underestimate overall levels consumption when compared to what is known about per capita consumption (Stockwell et al. 2004; WHO 2000), it is reasonable to assume that surveys which yield the greater overall estimated levels of consumption are, in fact, closer to actual levels of drinking (Stockwell et al. 2004). What is more, it is difficult to imagine that such 
a large proportion of respondents to the 1994 NDSHS routinely overestimated their consumption to such a large extent. It is more plausible that there was a significant degree of underestimation of actual consumption in the 2002 NATSISS. Adding to this, ABS reports on the results from its various surveys are often forthcoming about the limitations of the surveys. For instance, the 2002 NATSISS report states that 'results from previous ABS surveys and administrative data collections suggest a tendency for respondents to under-report substance use and alcohol and tobacco consumption levels' (ABS 2004c: 58). Perhaps, in this instance, some greater acknowledgment of the atypical nature of the methods and results should be at the forefront of any further discussion of the 2002 NATSISS.

Finally, the findings of the 2002 NATSISS are particularly questionable when Aboriginal risky/high-risk drinking is compared with Torres Strait Islander risky/high-risk drinking. The NATSISS found that a greater percentage of Torres Strait Islanders (nearly 22\%) were drinking in this way than were Aboriginal respondents (15\%). Although there are no specific alcohol and other drug surveys that have detailed Torres Strait Islander consumption patterns, observational and other data suggest that social drinking is the norm, rather than explosive, high-risk drinking (Torres Strait Health Workshop Working Party 1993: 15).

\section{Conclusions}

The questioning in the 2002 NATSISS on alcohol and tobacco use was neither confidential nor self-completed. Respondents were asked whether they would like a one-on-one interview but, in practice, they often answered questions in the presence of other family members. With alcohol and drug use being highly personal, potentially embarrassing and inevitably sensitive, and with family members perhaps listening in, it is hardly surprising that many respondents would have been unwilling to provide accurate estimates.

The findings on alcohol consumption do not only contradict the most reliable survey to date (the NDSHS), they run counter to qualitative understandings of Indigenous alcohol consumption, to smaller scale surveys, and counter to what we might call 'anthropological intuition' based on intimate daily participation in, and observations of, Indigenous life. Smaller scale studies such as these show that Indigenous people who drink do so at levels variously described as 'harmful', 'very heavy regular' or 'binge drinking'. These definitions have been used to describe remote communities (Hunter 1993; Martin 1998), rural towns (Perkins et al. 1994) and urban areas (Lake 1989; Tharawal Aboriginal Corporation 1994). All these report that moderate consumers were very much in the minority. They also consistently show an all-or-nothing pattern of consumption. Not only is 'moderate' consumption much less likely to be the chosen style, but there is active resistance to the idea of moderate or 'social' drinking among large sections of the Indigenous community. For the most part, we know that people tend to 
give up drinking altogether (i.e. become abstainers) rather than attempting (against huge social pressures) to consume moderately, and there are a number of good social and cultural reasons why Indigenous people make this choice (Brady 2004). It is highly unlikely that Indigenous drinking patterns would have changed so dramatically in the relatively short years since these studies were published.

What is of most concern, however, is the way in which findings from a survey such as the 2002 NATSISS quickly assume the mantle of authority. The new Productivity Commission report, Overcoming Indigenous Disadvantage (SCRGSP 2005: 22), has already cited the alcohol and other drug findings of the 2002 NATSISS, with its ink barely dry. Reproduced and reiterated in subsequent government reports and other documents, these statistics can take on the aura of authenticity, and fail to include the necessary caveats suggested by Biddle and Hunter in this volume - that is, to describe the findings on alcohol consumption specifically as referring to the 'Indigenous population living in private dwellings'. All future discussion of the 2002 findings should also acknowledge the atypical methods and results. The lack of corroborative evidence greatly reduces our confidence in these 2002 NATSISS findings and increases our concern that poor data have the potential to adversely affect funding priorities, program planning and future policy making. 